\section{Comparison of knee-high Mediven ulcer kit and Mediven Plus compression stockings: measurement of leg volume, interface pressure and static stiffness index changes}

\author{
Győző Szolnoky, Georgina Molnár, Dóra \\ Nemes-Szabó, Enikő Varga, Mónika \\ Varga, Lajos Kemény \\ Department of Dermatology and \\ Allergology, University of Szeged, \\ Szeged, Hungary
}

\section{Abstract}

Ulcer stockings are produced to have higher interface pressure and easier application compared to those of classic medical compression stockings. We aimed to compare volume decrease, pressure loss and stiffness index of a classical medical compression stocking and an ulcer stocking of the same interface pressure range in 10 patients with bilateral venous and 10 persons with lymphatic insufficiency. Interface pressure measurement in supine and standing positions and optoelectronic volumetry served for primary outcome variables. Both stockings were capable of inducing remarkable gradual volume reductions in different time points except classic stocking at 2 $\mathrm{h}$ in phleboedema care. Ulcer stocking pressures in lymph- and phleboedema were highly superior. In lymphedema a gradual interface pressure loss was attributed to both stockings regardless of body positions. Static stiffness indices did not differ statistically except classic stocking at baseline $(\mathrm{P}=0.0312)$ and $2 \mathrm{~h}$ $(\mathrm{P}=0.0082)$ comprising venous edema patients. Both stockings acted similarly but ulcer stocking had considerably higher interface pressures in each measurement and raised stiffness indices initially and the two-layer system facilitates donning therefore ulcer stocking could serve an alternative of classic medical compression stocking even in the treatment of leg edema.

\section{Introduction}

The intensive treatment of the two prevalent causes of chronic leg edema [chronic venous insufficiency (CVI) and lymphedema] is commonly based on various bandage systems. ${ }^{1}$ Inelastic bandages, especially when two or more are applied in an overlapping fashion, have high stiffness, a significant pressure loss is observed within the first hours of application due to the rapid volume reduction. ${ }^{2}$ Medical compression stockings (MCSs) are elastic devices with relatively low stiffness index $(<10) .{ }^{3}$ Unlike bandages, MCSs are observed to loose original interface pressure to a lesser degree. ${ }^{2}$ There is an emerging body of evidence that MCSs are also capable of efficient volume reduction even in the intensive therapeutical phase. ${ }^{1}$ Taken the previous data together, MCSs are presumed to possess some important features of efficient compression, however the achievable high interface pressures may associate low patient compliance as both donning and removal of the garment cause difficulties and require outstandingly high forces. ${ }^{4}$ The pressure of the two superimposed stockings was shown to roughly correspond to the direct addition of the interface pressure exerted by the single layer due to interface friction. ${ }^{5}$ Overlapping stockings efficiently raise interface pressure and alleviate application. Classic MCSs are recommended for daily use but depending on interface pressure in supine position, patients are sometimes asked to wear their stockings overnight. The nocturnal wear of understocking of ulcer garments is preferred. ${ }^{5}$ The new generation of stockings with double-layers is preferably recommended for leg ulcer healing but its potential advances over traditional MCSs give rise to a comparative study in view of stiffness.

This was the background for a clinical study in which we compared interface pressures of an ulcer stocking with that of a traditional MCS belonging to the identical pressure range.

\section{Materials and Methods}

A total of 20 legs from ten out-patients with bilateral CVI [three males, seven females; age $52-75$, median 61 ; mean body mass index (BMI) (kg/m²): 30.57 (21.52-45.84); mean disease duration (years): 5 (1-20); clinical Clinical-Etiology-Anatomy-Pathophysiology classification (CEAP)-classes C3-6] and another 20 legs of ten secondary lymphedema outpatients with bilateral lower limb affections (three males, seven females; age 55-81, median 70; mean BMI $\left(\mathrm{kg} / \mathrm{m}^{2}\right): 36$ (24-41); mean disease duration (years): 8 (4-14) were recruited. Each of the bilateral secondary lymphedema cases was stage II comprising 5 persons with gynecological cancer treatmentrelated moderate lymphedema and another 5 patients where repeated erysipelas affecting both legs at different time caused lymphedema. CVI was diagnosed using color-coded duplex ultrasonography. Patients did not wear any form of compression garment $48 \mathrm{~h}$ before the beginning of the trial (wash-out period) and lymphedematous legs did not receive sup-
Correspondence: Győző Szolnoky, Department of Dermatology and Allergology, University of Szeged, 6720 Szeged, Korányi fasor 6., Hungary. E-mail: szolnoky@dermall.hu

Key words: volumetry, static stiffness index, medical compression stocking, ulcer stocking.

Conference presentation: part of this paper was presented at the International Compression Club (ICC) Meeting on Stiffness of Compression Devices, 2012 May 25, Vienna, Austria (http:// www.icc-compressionclub.com/).

Acknowledgments: Medi (Bayreuth, Germany) Company supported the clinical study with standard Mediven Plus ccl $3 \mathrm{AD}$ and Mediven ulcer kit stockings.

Received for publication: 17 January 2013.

Revision received: 8 March 2013.

Accepted for publication: 4 April 2013.

This work is licensed under a Creative Commons Attribution 3.0 License (by-nc 3.0).

(C) Copyright G. Szolnoky et al., 2013

Licensee PAGEPress, Italy

Veins and Lymphatics 2013; 2:e13

doi:10.4081/vl.2013.e13

plementary treatment (e.g. manual lymph drainage, intermittent pneumatic compression). Inclusion criteria were in accordance with the recommendations of the International Compression Club. ${ }^{1}$ Informed consent was obtained from each patient and the study protocol conformed to the regular ethical guidelines, as reflected in a prior approval by the University of Szeged human research committee. According to limb girths standard belowknee stockings (Mediven ulcer kit and Mediven Plus compression class 3) were provided by the Medi Company (Bayreuth, Germany). Interface pressure was measured by Kikuhime (Medi Trade, Soro, Denmark) device ${ }^{6}$ using small pressure probe placed to point B1 at baseline, 2, 4 and $24 \mathrm{~h}$ in standing and supine positions, as well. Pressure probe was not held continuously under the stocking but was placed immediately after pulling down the compression material then stocking was redone and finally the measurement was completed. According to our standards, Mediven ulcer kit was assigned to right, while Mediven Plus to the left leg. Stockings were worn for 24 $h$ with a surprisingly sufficient tolerability. The static stiffness index (SSI) was calculated as the difference between standing and supine pressures. ${ }^{7}$ Leg volumes were assessed with infrared optoelectronic measurement using Perometer (Perimed, Wuppertal, Germany) ${ }^{8}$ at baseline and immediately after pulling down the stockings taking only 2-4 min in each case. 
Feet and calves were subjected to volumetry. To compare SSI of the two products (right side $v s$ left side), the non-parametric Mann-Whitney test was used. Comparisons between the pressure and volume values at different time points using the same type of stocking were made by Wilcoxon signed rank test as a nonparametric measure. $P$ values lower than 0.05 were considered as statistically significant.

\section{Results}

\section{Volume change}

Both Mediven ulcer kit and Mediven Plus stockings were capable of inducing remarkable gradual volume reductions in different time points. Each of the measured volume decreases appeared to be significant except Mediven Plus at $2 \mathrm{~h}$ among patients with phleboedema.

\section{Interface pressure}

The pressure exerted by ulcer stocking in lymph- and phleboedema was highly superior to that of Mediven Plus at each measurement in lying and standing positions except a single assessment at $2 \mathrm{~h}$ in upright position $(\mathrm{P}=$ 0.0707) of the patients with lymphedema.

\section{Pressure alteration}

In lymphedema a gradual interface pressure loss was attributed to both compression stockings regardless of body position positions (Figure 1). Mediven Plus failed to cause a pressure decrease $2 \mathrm{~h}$ after the beginning of application in supine $(\mathrm{P}=0.1016)$ and standing $(\mathrm{P}=0.509)$ positions, as well. Venous edema treatment with Mediven Plus associated significant pressure losses in supine positions but did not provoke any significant changes of interface pressures $(\mathrm{P}=0.0762$ at $2 \mathrm{~h}$, $\mathrm{P}=0.1602$ at $4 \mathrm{~h}$ and finally $\mathrm{P}=0.0547$ at $24 \mathrm{~h}$ ) in standing posture (Figure 2).

\section{Static stiffness index}

The calculated static stiffness indices did not differ statistically regardless of compression material in lymphedema (Figure 3A), however this parameter of Mediven Plus (median: 2.00) was significantly inferior to that of ulcer stocking (median: 4.00) at the first two measurements (baseline: $\mathrm{P}=0.0312$ and $2 \mathrm{~h}: \mathrm{P}=0.0082$ ) comprising venous edema patients (Figure 3B).

\section{Discussion}

According to experiments in the field of compression therapy two factors play a pivotal role in setting an efficient therapy. Interface
A

Ulcer kit interface pressures in supine position (lymphedema)

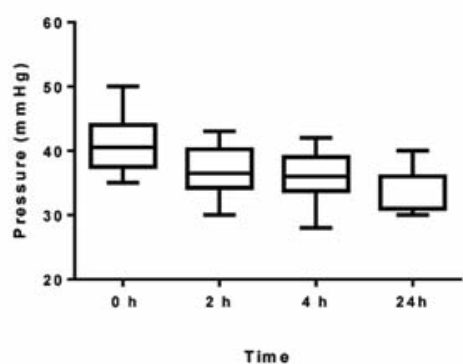

C Ulcer kit interface pressures in supine position (lymphedema)

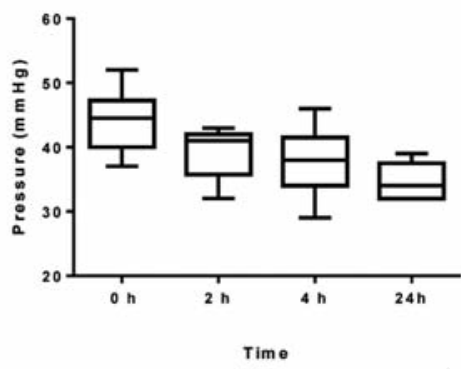

B

Mediven interface pressures in supine position (lymphedema)

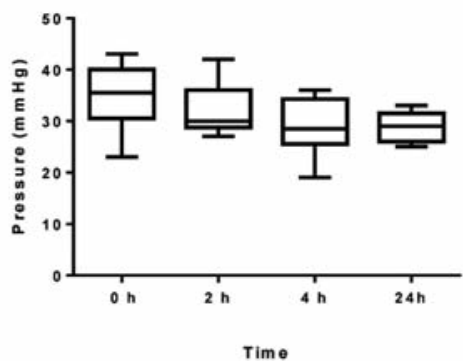

D Mediven interface pressures in standing position (lymphedema)

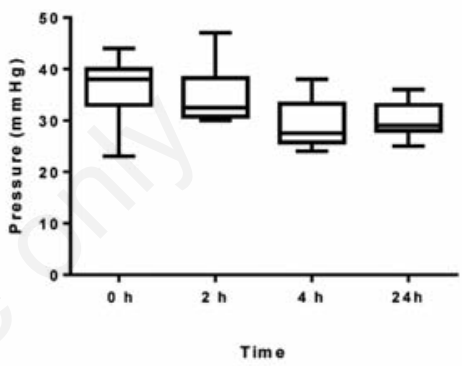

Figure 1. Interface pressures of Mediven Ulcer kit and Mediven Plus AD ccl 3 stockings in supine $(A, B)$ and standing $(C, D)$ positions in lymphedema.

A
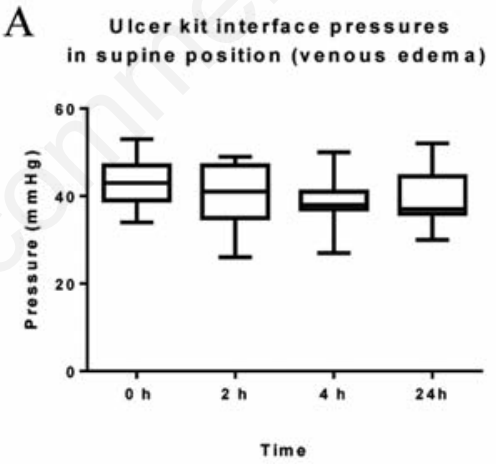

C Ulcerkit interface pressures in standing position (venous edema)

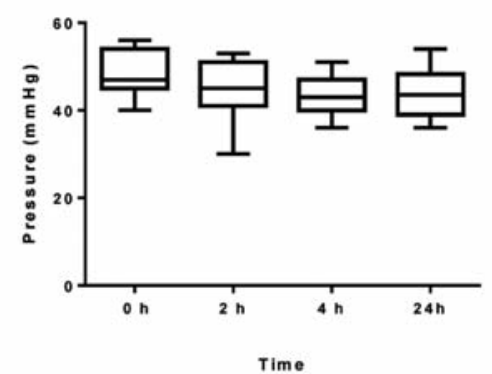

B

Mediven interface pressures in supine position (venous edema)

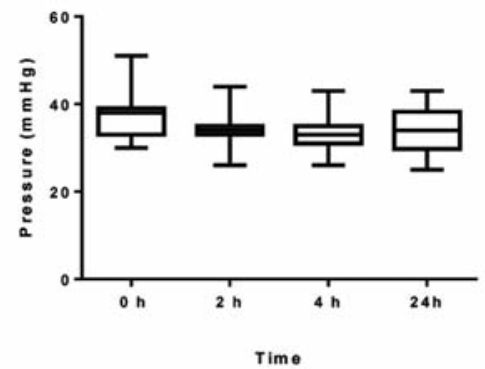

D
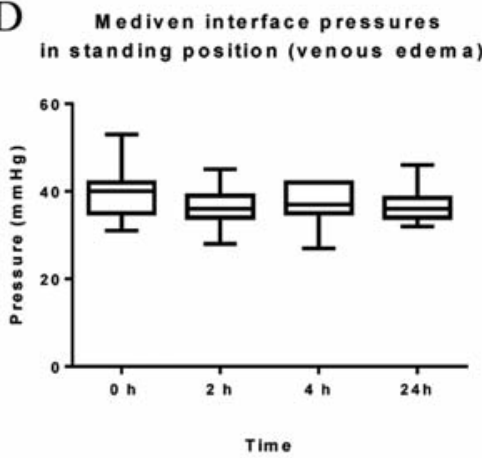

Figure 2. Interface pressures of Mediven Ulcer kit and Mediven Plus AD ccl 3 stockings in supine $(A, B)$ and standing $(C, D)$ positions in venous edema. 
pressure measured under the compression material is able to restore venous and lymphatic insufficiency along narrowing veins and ameliorating interstitial pressure. Clinical studies demonstrated that the application of higher external pressure lead to a faster venous leg ulcer healing by efficiently counteracting the high ambulatory venous pressure thus providing an enhanced venous flow. Lower extremity edema sometimes presents in a combined form including impaired venous function, lymphatic insufficiency and increased capillary permeability, ${ }^{1}$ thus the relatively high edema volume warrants fairly high external pressure. Evacuation of edema and the most advanced form of venous insufficiency are preferably directed to inelastic compression bandaging in a multilayer and multicomponent fashion where interface pressure is strongly correlated with the tensile forces. ${ }^{9,10}$ MCSs are usually used in the maintenance phase where achieved results (volume decrease, healed ulcer) should be preserved. ${ }^{1}$ The higher success rate in leg affection is attributed to the applied pressure. If the external pressure meets the ordinary range of ambulatory venous pressure in impaired function it might minimize the risk of recurrence. Medical compression stockings are manufactured from elastic material so as to facilitate donning and positioning over bony prominences but the elastic properties possess at least two disadvantages: stockings less efficiently assist muscle pump ${ }^{11}$ and keep nearly the same pressure regardless of position having low SSIs. A major burden of increasing compression pressure is the tolerability. Classic medical compression stockings of higher pressures cause difficulties in donning and keeping them on legs in lying position. A new generation of stockings tends to alleviate these problems comprising an under- and an overstocking with relatively low pressures. These two superimposed garments set the final pressure. ${ }^{5}$ These overlapping stockings were designed especially to treat leg ulcers but their other advantages made them interesting for other objectives like the use in chronic leg edema (e.g. phleb- or lymphedema) however remained relatively poorly characterized and compared to other compression materials. Beyond their relatively easy application we were able to experience that ulcer stockings also exerted significant leg volume reduction and brought up significantly higher interface pressure compared to classical MCSs. The difference between lying and standing positions results SSI that is an accurate indicator of appropriate stocking selection for patients. ${ }^{12}$ Low pressure at supine position and a substantial rise after standing up provides a comfortable wear and an efficient prevention against edema formation and venous dilation. We were able to show that the SSI of the given ulcer stocking was able to exceed that of compression class 3 medical stocking in venous edema during the initial phase of treatment however it remained still relatively low. Stockings with higher stiffness have a higher anti-edematous efficacy. ${ }^{13}$ A previous clinical trial disclosed that the superposition of two stockings did not only increase the interface pressure, but had a further additive effect to the stiffness of the final stocking combination. ${ }^{3}$

To our knowledge this is the first comparative study examining two types of stockings from the aspect of stiffness index as one of the most emphasized primary outcome variable. From the practical point of view we recommend the use of ulcer stockings instead of classic MCS with identical interface pressure when the final pressure should be adjusted along easier doffingand in case of distinct leg edema forms.
A Static stiffness index in lymphedema

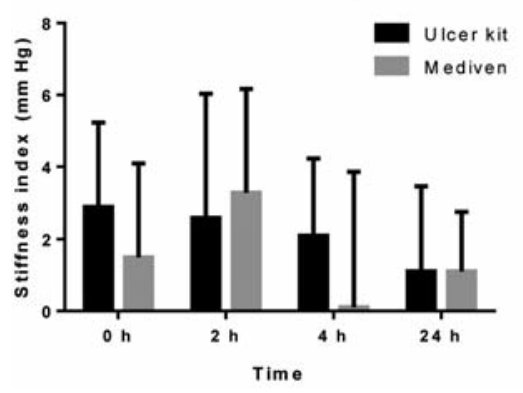

B

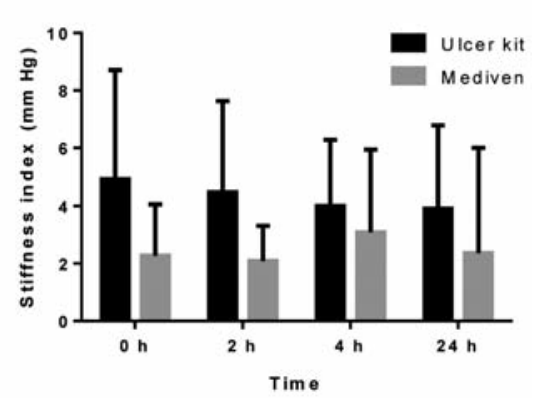

\section{References}

1. Stout N, Partsch H, Szolnoky G, et al. Chronic edema of the lower extremities: international consensus recommendations for compression therapy clinical research trials. Int Angiol 2012;31:316-29.

2. Larsen AM, Futtrup I. Watch the pressure it drops! EWMA J 2004;4:8-12.

3. Partsch H, Partsch B, Braun W. Interface pressure and stiffness of ready made compression stockings: comparison of in vivo and in vitro measurements. J Vasc Surg 2006;44:809-14.

4. Willenberg T, Lun B, Amsler F, Baumgartner I. Ease of application of medical compression-stocking systems for the treatment of venous ulcers. Eur J Vasc Endovasc Surg 2010;40:129-13.

5. Partsch B, Partsch H. Compression stockings for treating venous leg ulcers: measurement of interface pressure under a new ulcer kit. Phlebology 2008;23:40-6.

6. Flaud P, Bassez S, Counord JL. Comparative in vitro study of three interface pressure sensors used to evaluate medical compression hosiery. Dermatol Surg 2010; $36: 1930-40$.

7. Partsch H. The static stiffness index. A simple method to assess the elastic property of compression material in vivo. Dermatol Surg 2005;31:625-30.

8. Pannier F, Rabe E. Optoelectric volume measurements to demonstrate volume changes in the lower extremities during orthostasis. Int Angiol 2010;29:395-400.

9. Partsch H, Flour M, Smith PC; International Compression Club. Indications for compression therapy in venous and lymphatic disease consensus based on experimental data and scientific evidence. Under the auspices of the IUP. Int Angiol 2008;27:193-219.

10. Partsch H, Clark M, Mosti G, et al. Classification of compression bandages: practical aspects. Dermatol Surg 2008;34: 600-9.

11. Mosti G, Partsch H. Measuring venous pumping function by strain-gauge plethysmography. Int Angiol 2010;29:421-5.

12. Van der Wegen-Franken K, Tank B, Neumann M. Correlation between the static and dynamic stiffness indices of medical elastic compression stockings. Dermatol Surg 2008;34:1477-85.

13. Van Geest AJ, Veraart JC, Nelemans P, Neumann HA. The effect of medical elastic compression stockings with different slope values on edema. Measurements underneath three different types of stockings. Dermatol Surg 2000;26:244-7.
Figure 3. Comparison of static stiffness indices of Mediven Ulcer kit and Mediven Plus $\mathrm{AD}$ ccl 3 stockings in lymphedema (A) and venous edema (B). 